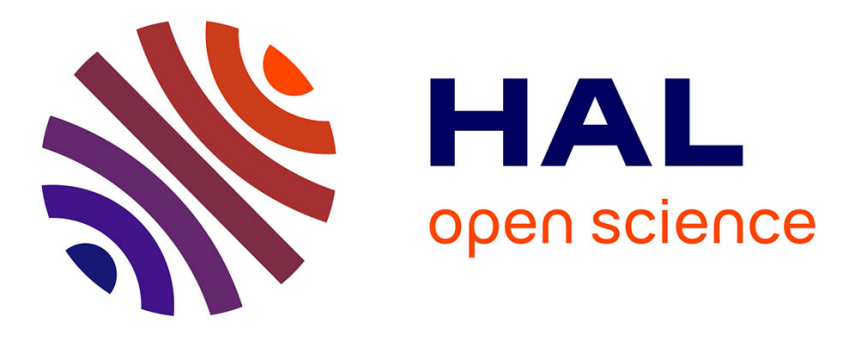

\title{
Synthesis of aminopyrimidylindoles structurally related to meridianins
}

\author{
Emilie Rossignol, Ali Youssef, Pascale Moreau, Michelle Prudhomme, Fabrice
}

Anizon

\section{- To cite this version:}

Emilie Rossignol, Ali Youssef, Pascale Moreau, Michelle Prudhomme, Fabrice Anizon. Synthesis of aminopyrimidylindoles structurally related to meridianins. Tetrahedron, 2007, 63, pp.10169-10176. 10.1016/j.tet.2007.07.095 . hal-00170736

\section{HAL Id: hal-00170736 https://hal.science/hal-00170736}

Submitted on 10 Sep 2007

HAL is a multi-disciplinary open access archive for the deposit and dissemination of scientific research documents, whether they are published or not. The documents may come from teaching and research institutions in France or abroad, or from public or private research centers.
L'archive ouverte pluridisciplinaire HAL, est destinée au dépôt et à la diffusion de documents scientifiques de niveau recherche, publiés ou non, émanant des établissements d'enseignement et de recherche français ou étrangers, des laboratoires publics ou privés. 


\title{
Synthesis of aminopyrimidylindoles structurally related to meridianins
}

\author{
Emilie Rossignol, Ali Youssef, Pascale Moreau, Michelle Prudhomme and Fabrice Anizon*
}

Université Blaise Pascal, SEESIB-UMR CNRS 6504, 24 Avenue des Landais, 63177 Aubière Cedex, France

\begin{abstract}
The synthesis of new meridianin derivatives substituted at the C-5' position of the 2-aminopyrimidine ring by various aryl groups and substituted or not by a methyl group on the indole nitrogen is described. The 2-aminopyrimidine ring was obtained via a Bredereck synthesis. Aryl groups were introduced by Suzuki cross-coupling after bromination of the 2 -aminopyrimidine ring at the C-5' position.
\end{abstract}

\section{Introduction}

Many kinase inhibitors from natural origin are sources of inspiration for the discovery of new biologically active compounds. A large number of nitrogen aromatic heterocycles containing an indole or a carbazole framework exhibit kinase inhibitory properties. Granulatimide and isogranulatimide, carbazoles isolated from the ascidian Didemnum granulatum, and structurally related analogs are Checkpoint kinase 1 (Chk1) inhibitors (Fig. 1). ${ }^{1,2}$ Purine derivatives like roscovitine and olomoucine, two compounds isolated from starfish oocytes, are potent cyclin-dependent kinase inhibitors. ${ }^{3}$ Indirubin, the active ingredient of the Chinese preparation Danggui Longhui Wan used to treat chronic diseases, indirubin derivatives, ${ }^{4,5}$ as well as indolocarbazole bacterial metabolites staurosporine, ${ }^{6,7} \mathrm{UCN}-01,{ }^{8}$ and $\mathrm{K} 252-\mathrm{c}^{9}$ are also described as potent kinase inhibitors. Synthetic compounds such as bisindolylmaleimides ${ }^{10}$ and 4-aryl-3-indolylmaleimides ${ }^{11,12}$ are known kinase inhibitors (Fig. 1).

Meridianin alkaloids, which were isolated and characterized from the south atlantic tunicate Aplidium meridianum, ${ }^{13}$ are indole derivatives substituted at the $\mathrm{C}-3$ position by a 2-aminopyrimidine ring. Meridianins $A-G^{13-15}$ (Fig. 1) were described as potent kinase inhibitors ${ }^{15}$ and some derivatives displayed antitumor activity. ${ }^{16}$

In the course of the synthesis of new kinase inhibitors, we were interested in meridianin derivatives substituted at the ${\mathrm{C}-5^{\prime}}^{\prime}$ position of the 2-aminopyrimidine ring. Various

Keywords: Meridianins; Kinase inhibitors; Pyrimidine; Indole; Antitumor agents.

* Corresponding author. Fax: +33 4734077 17; e-mail: Fabrice. ANIZON@univ-bpclermont.fr 3-(2-aminopyrimidin-4-yl)-indoles substituted at the C-5' and C- $6^{\prime}$ positions and/or on the amino group of the 2-aminopyrimidine ring have been described in the literature. ${ }^{16-37}$ Compounds substituted at $\mathrm{C}-5^{\prime}$ position by cyano, carbohydrazonamido, carboxy, and methyl groups or by a chlorine or a fluorine atom have previously been reported (Fig. 1). ${ }^{16,17,19}$ These recent publications prompted us to report our own results. Substituent at the C-5 position appeared to be important in terms of biological activity. Indeed, we observed a dramatic increase of kinase inhibitory properties when the $\mathrm{C}^{-} 5^{\prime}$ position of meridianin $\mathrm{G}$ was substituted by a bromine atom (Scheme 1 and Table 1). The kinase activities of compounds 3 (meridianin G) and $\mathbf{5}$ toward eight protein kinases were evaluated by Upstate's kinase profiler screening service (Dundee, Scotland). Both compounds were tested at a compound concentration of $10 \mu \mathrm{M}$ under standard conditions determined by Upstate ${ }^{38-40}$ for each selected kinase (MKK1, ERK2, RSK2, PKC- $\alpha$, GSK3- $\beta$, CDK2/A, CK2, and MST2-Table 1). Compound 5 exhibited a significantly higher inhibitory activity than meridianin G (compound 3) against all the kinases tested. Indeed, compound 5 inhibits all the kinases tested with percentages of inhibition over $80 \%$, and is particularly potent toward MKK1 and MST2 (percentage of inhibition higher than $95 \%$ ), whereas the inhibitory properties of compound 3 were found to be much lower, with percentages of inhibition from $9 \%$ (RSK2) to $66 \%$ (MKK1). These results encouraged us to synthesize meridianin derivatives bearing other substituents at the C-5 $5^{\prime}$ position. Since the late $1980 \mathrm{~s}^{11,12}$ and recently, ${ }^{41}$ it was reported that aryl groups at the C-4 position of 3-indolylmaleimides led to compounds exhibiting interesting kinase inhibitory properties. Therefore, we decided to introduce aryl rings at the $\mathrm{C}-5^{\prime}$ position of the pyrimidine ring of meridianin $\mathrm{G}$. Compounds bearing an aryl group at the C-6' position were reported, ${ }^{22,25}$ but to our knowledge, 

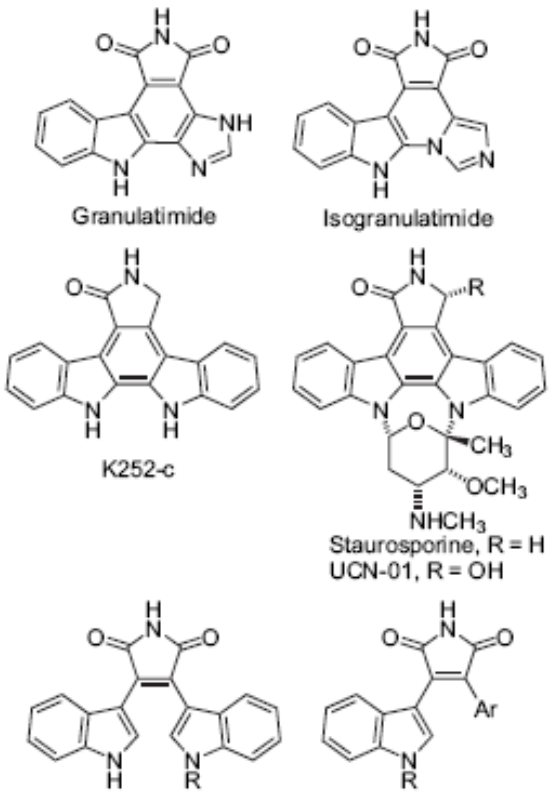

bisindolylmaleimides

4-Aryl-3-indolylmaleimides
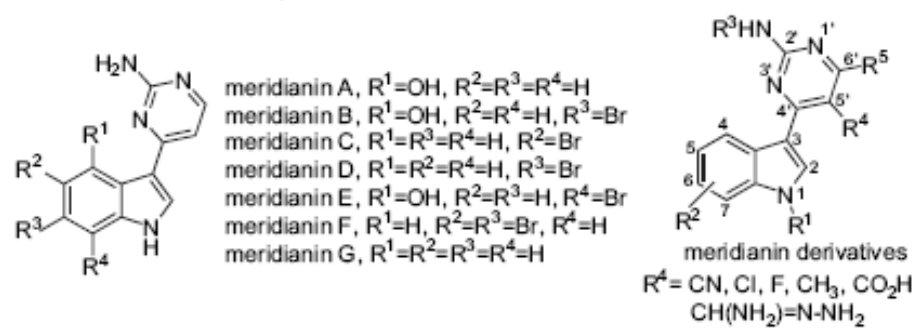

Figure 1. Kinase inhibitors and meridianin derivatives described in the literature.

meridianin derivatives with an aryl group at the $\mathrm{C}-5^{\prime}$ position have never been described. The replacement of the maleimide ring of 4-aryl-3-indolylmaleimides previously mentioned by the 2 -aminopyrimidine ring could reinforce the interaction inside the ATP-binding pocket of the target kinase. To get an insight into the importance of the $\mathrm{NH}$ of the indole moiety, we also prepared derivatives substituted on the indole nitrogen with a methyl group. The introduction

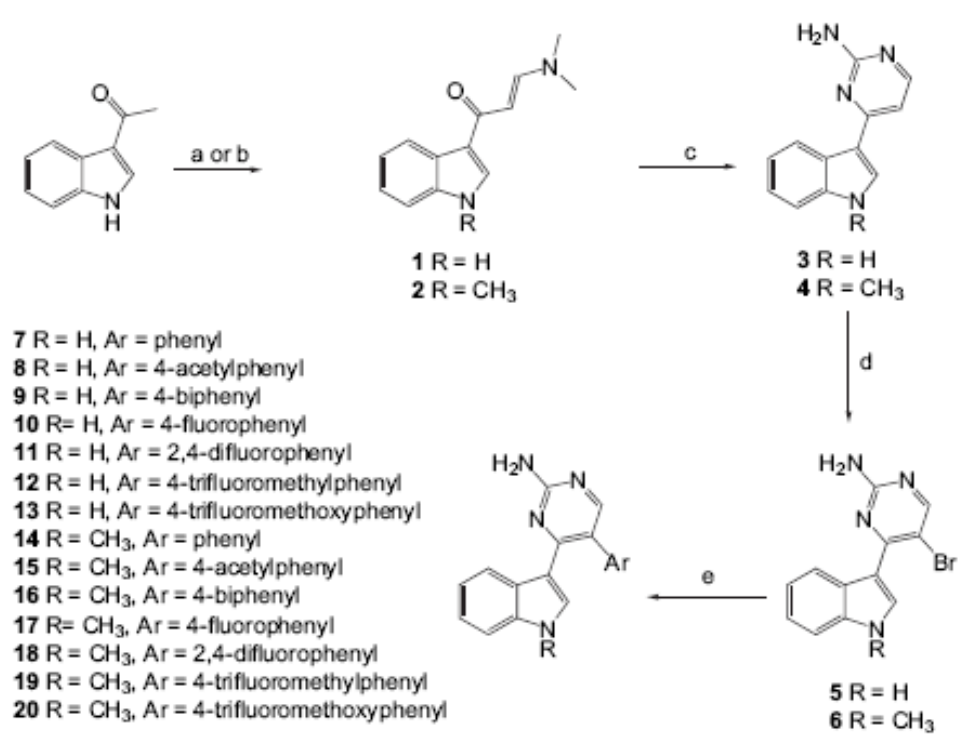

Scheme 1. Synthesis of meridianin derivatives. (a) DMF/DMF-di-tert-butylacetal, reflux, $\mathrm{R}=\mathrm{H}$; (b) DMF/DMF-DMA, reflux, $\mathrm{R}=\mathrm{CH}_{3}$; (c) iso-propanol, gua-

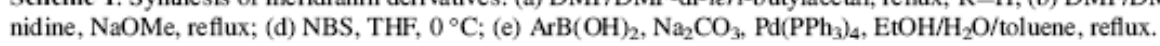


Table 1. Percentage of inhibition of various kinases at a compound concentration of $10 \mu \mathrm{M}$, for compounds 3 and 5

\begin{tabular}{lllllllll}
\hline \multicolumn{1}{l}{ Compound } & MKK1 & ERK2 & RSK2 & PKC- $\alpha$ & GSK3- $\beta$ & CDK2/A & CK2 & MST2 \\
\hline $\mathbf{3}$ & 66 & 36 & 9 & 56 & 43 & 54 & 38 & 31 \\
5 & 98 & 92 & 85 & 91 & 87 & 85 & 93 & 96
\end{tabular}

MKK1, Mitogen-activated Kinase Kinase 1; ERK2, Extracellular signalRegulated Kinase 2; RSK2, p90 ribosomal S6 kinase 2; PKC- $\alpha$, Protein Kinase C- $\alpha$; GSK3- $\beta$, Glycogen Synthase $3-\beta$; CDK2/A, Cyclin-Dependent Kinase $2 /$ cyclin A; CK2, Casein Kinase 2; MST2, Mammalian STE20like kinase 2.

of an alkyl group at this position could be relevant for biological activity, as it was shown in bisindolylmaleimide series. ${ }^{10,42}$

\section{Chemistry}

Meridianins have previously been synthesized from indole derivatives. The first of the three reported approaches consisted in a Suzuki cross-coupling with indole-3-boronic acid derivatives. ${ }^{43} \mathrm{~A}$ Bredereck synthesis was also described from $\beta$-enaminones, which were, in turn, obtained from 3acetylindole derivatives. ${ }^{16,44,45}$ More recently, meridianins were obtained from trimethylsilylynones indole derivatives. ${ }^{46}$ Commercially available 3 -acetylindole was the starting point of our synthesis, which gave access to the meridianin analogs $\mathbf{5}$ and $\mathbf{6}$ in a straightforward three-step synthesis following the Bredereck approach (Scheme 1). ${ }^{16,44,45,47}$ While the reported Bredereck routes started from $\mathrm{N}$-tosylated 3-acetylindole, our synthesis was initiated with non-protected 3-acetylindole. This allowed us to obtain both meridianins 5 and $\mathbf{6}$ with the indole nitrogen methylated or simply protonated without the need for protection-deprotection steps. When 3-acetylindole was treated with DMF/ dimethylformamide-dimethylacetal (DMF-DMA), ${ }^{48} \mathrm{~N}$ methylated compound 2 was obtained in $85 \%$ yield. Using DMF/dimethylformamide-di-tert-butylacetal instead of DMF/DMF-DMA led to compound $\mathbf{1}$ in $71 \%$ yield. When refluxing in toluene/DMF-DMA, compound 1 was obtained in only $24 \%$ yield but no $N$-methylated compound 2 was observed. Increasing the reflux temperature by replacement of toluene by xylene led to compound 2 in a poor yield.

The $E$ configuration of the enaminone double bond in $\mathbf{1}$ and $\mathbf{2}$ was determined by NMR spectrometry. From the coupling constant between the two ethylenic protons $(J=12.5 \mathrm{~Hz})$, configuration could not be unambiguously determined. Therefore, a 2D-NOESY NMR experiment was performed on compound 1 (Fig. 2). The similar NOE effects between each two ethylenic protons at $5.77 \mathrm{ppm}$ and $7.53 \mathrm{ppm}$ and the $\mathrm{CH}_{3}$ groups showed that compound $\mathbf{1}$ has an $E$ configuration. We confirmed the $E$ configuration by the measurement of the ${ }^{3} J\left({ }^{1} \mathrm{H},{ }^{13} \mathrm{C}=\mathrm{O}\right)$ coupling constant existing between the ethylenic proton at the $\beta$-position relative to the carbonyl group and the ${ }^{13} \mathrm{C}$ of the ketone function. Indeed, the configuration of the double bond of $\alpha, \beta$-unsaturated carbonyl compounds can be determined on the basis of this long range heteronuclear coupling constant. The ${ }^{3} J(\mathrm{H}, \mathrm{C})$ between ${ }^{1} \mathrm{H}$ and ${ }^{13} \mathrm{C}=\mathrm{O}$ nuclei with a $\mathrm{Z}$ orientation around the double bond are usually smaller $(2-6 \mathrm{~Hz})$ than those with an $E$ orientation $(8-12 \mathrm{~Hz}){ }^{49,50}$ Thus, in our case, the measured ${ }^{3} J(\mathrm{H}, \mathrm{C})$ value of $4 \mathrm{~Hz}$ indicated the $E$ configuration for

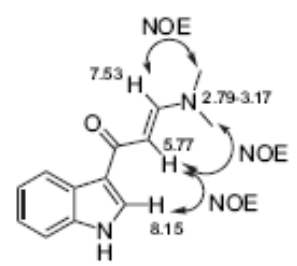

Figure 2. Determination of the configuration of the $\beta$-enaminone double bond of compound 1 by 2D-NOESY NMR experiment.

$\beta$-enaminone double bond of compound 1. Surprisingly, in the ${ }^{1} \mathrm{H}$ NMR spectra of enaminones 1 and 2 in DMSO- $d_{6}$ or in $\mathrm{CDCl}_{3}$, the two methyl groups of the $\beta$-enaminone moiety appeared as a broad signal between $2.79 \mathrm{ppm}$ and $3.17 \mathrm{ppm}$ for 1 and between $2.85 \mathrm{ppm}$ and $3.09 \mathrm{ppm}$ for 2 . The ${ }^{1} \mathrm{H}$ and ${ }^{13} \mathrm{C}$ NMR spectra of structurally related compounds ${ }^{16,45}$ reported in the literature showed two independent peaks for each methyl of the $\mathrm{N}\left(\mathrm{CH}_{3}\right)_{2}$ group (Fig. 3). This suggests that the rotation energy barrier around the $=\mathrm{C}-\mathrm{N}$ bond is sufficient to make the $\mathrm{N}$-methyl groups magnetically non-equivalent. This high rotation energy could be explained by the partial $\pi$ character of the $\mathrm{C}-\mathrm{N}$ bond, part of the conjugated $\beta$-enaminone system. In the case of compounds 3 and $\mathbf{4}$, the absence of electron-withdrawing protecting group potentially decreases the $\pi$ character of the $\mathrm{C}-\mathrm{N}$ bond. This would lower the rotation energy barrier and provoke coalescence of the two $\mathrm{CH}_{3}$ peaks. Consequently, the two methyl groups of the $\beta$-enaminone moiety were not visible on the $1 \mathrm{D}^{13} \mathrm{C}$ NMR in DMSO- $d_{6}$ or in $\mathrm{CDCl}_{3}$. Nevertheless, ${ }^{1} \mathrm{H}-{ }^{13} \mathrm{C}$ HSQC experiment showed the correlation between these ${ }^{1} \mathrm{H}$ broad signal and two carbons.

Compounds 1 and $\mathbf{2}$ were treated with guanidine in iso-propanol in the presence of sodium methoxide to give compounds 3 and $\mathbf{4}$ in $74 \%$ and $44 \%$ yield, respectively. Meridianins 3 and $\mathbf{4}$ were then brominated with $\mathrm{N}$-bromosuccinimide (NBS) in THF. Compounds 5 and $\mathbf{6}$ were obtained in $95 \%$ and $94 \%$ yield, respectively. The electrophilic substitution by the bromine atom was clearly directed to the 5-position of the pyrimidine ring. ${ }^{51}$ The position of the bromine atom was unambiguously determined by NMR. ${ }^{1} \mathrm{H}$ and ${ }^{13} \mathrm{C}$ NMR signals were assigned from $1 \mathrm{D}$ and $2 \mathrm{D}\left(\operatorname{COSY}{ }^{1} \mathrm{H}-{ }^{1} \mathrm{H}, \mathrm{HSQC}{ }^{1} \mathrm{H}-{ }^{13} \mathrm{C}\right.$, and $\left.\mathrm{HMBC}{ }^{1} \mathrm{H}-{ }^{13} \mathrm{C}\right)$ experiments. The two aromatic protons of the pyrimidine ring of compound 4 correspond to two doublets at $6.94 \mathrm{ppm}$ for $\mathrm{H}-5^{\prime}$ and at $8.10 \mathrm{ppm}$ for H-6'. After bromination of 3, only one singlet corresponding to $\mathrm{H}-6^{\prime}$ was observed at $8.31 \mathrm{ppm}$. The ${ }^{13} \mathrm{C}$ NMR spectra of compounds 4 and $\mathbf{6}$ also show the low field methine carbon corresponding to C-6 at $157.1 \mathrm{ppm}$ and $159.5 \mathrm{ppm}$, respectively.

Suzuki cross-couplings were then performed between brominated compound 5 or 6 and substituted phenylboronic

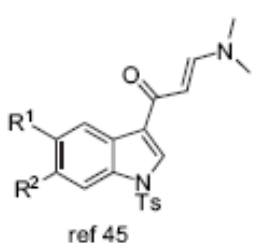

ref 45

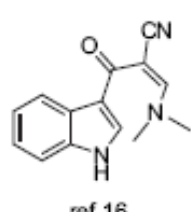

ref 16
Figure 3. Examples of the literature in which the two methyl groups of $\mathrm{N}\left(\mathrm{CH}_{3}\right)_{2}$ appeared as distinct signals in the ${ }^{1} \mathrm{H}$ NMR spectra. 
acids. Phenyl group of boronic acids was variously substituted by either halogen atoms (compounds 10, 11, 17, and 18), aryl ( 9 and 16), acetyl (8 and 15), trifluoromethyl (12 and 19), and trifluoromethoxy (13 and 20) groups. Couplings were carried out using palladium tetrakis(triphenylphosphine) in a $1: 1: 1$ toluene/EtOH/ $\mathrm{H}_{2} \mathrm{O}$ mixture in the presence of sodium carbonate, ${ }^{52}$ leading to compounds $\mathbf{7 - 2 0}$ in $21-60 \%$ isolated yields.

\section{Conclusion}

Encouraging preliminary biological evaluation of brominated derivative 5 led us to synthesize new meridianin derivatives. The synthetic approach described here allowed the substitution at the $\mathrm{C}-5^{\prime}$ position of the pyrimidine moiety by diversely substituted phenyl rings (compounds 7-20). Moreover, since in bisindolylmaleimide series substitution of the indole nitrogen with an alkyl group led to potent kinase inhibitors, compounds 14-20, substituted on the indole nitrogen by a methyl group, were also prepared. The biological evaluation of compounds $\mathbf{7 - 2 0}$ is currently in progress.

\section{Experimental}

\subsection{General}

IR spectra were recorded on a Perkin-Elmer Paragon 500 spectrometer $\left(\bar{\nu}_{\max }\right.$ in $\left.\mathrm{cm}^{-1}\right)$. NMR spectra were recorded on a Bruker AVANCE $400\left({ }^{1} \mathrm{H}: 400 \mathrm{MHz},{ }^{13} \mathrm{C}: 100 \mathrm{MHz}\right)$ or AVANCE $500\left({ }^{1} \mathrm{H}: 500 \mathrm{MHz},{ }^{13} \mathrm{C}: 125 \mathrm{MHz}\right)$; chemical shifts $\delta$ are noted in parts per million and the following abbreviations are used: singlet (s), doublet (d), triplet (t), quadruplet (q), doubled doublet (dd), doublet of doublet of doublet (ddd), doubled triplet (dt), multiplet (m), broad signal (br s). Mass spectra (ESI ${ }^{+}$) were recorded on a high resolution Waters Micro Q-Toff apparatus. Chromatographic purifications were performed on flash silica gel Geduran SI 60 (Merck) $0.040-0.063 \mathrm{~mm}$ column chromatography. TLC were performed on fluorescent silica gel plates $(60$ F254 from Merck).

\subsection{Synthesis}

4.2.1. 3-(3- $N, N$-Dimethylamino-1-oxoprop-2-enyl)-1Hindole 1. Dimethylformamide-di-tert-butylacetal $(2.4 \mathrm{~mL}$, $10.0 \mathrm{mmol}$ ) was added to a solution of 3-acetylindole (318 mg, 2.0 mmol) in DMF (6 mL) and the solution was refluxed for $48 \mathrm{~h}$. Brine $(10 \mathrm{~mL})$ was added to the reaction mixture. After extraction with $\mathrm{CH}_{2} \mathrm{Cl}_{2}(3 \times 10 \mathrm{~mL})$, the combined organic fractions were dried over $\mathrm{MgSO}_{4}$ and concentrated under vacuum. The residue was dissolved in EtOAc $(5 \mathrm{~mL})$ and precipitated with cyclohexane $(15 \mathrm{~mL})$. The precipitate was collected by filtration and washed with $\mathrm{Et}_{2} \mathrm{O}$ $(3 \times 10 \mathrm{~mL})$ to give $1(303 \mathrm{mg}, 1.41 \mathrm{mmol}, 71 \%$ yield) as a pale yellow powder.

Mp 219-221 ${ }^{\circ} \mathrm{C}$. IR (KBr) 3448, 1630, 1508, 1446, 1416, $1376,1304,1284,1164,1114,1095,1011$. MS m/z (EI) $214\left(\mathrm{M}^{+}\right) .{ }^{1} \mathrm{H}$ NMR $\left(400 \mathrm{MHz}, \mathrm{DMSO}-d_{6}\right): 2.79-3.17(6 \mathrm{H}$, br s, $\left.\mathrm{CH}_{3}\right), 5.77(1 \mathrm{H}, \mathrm{d}, J=12.5 \mathrm{~Hz}), 7.06-7.16(2 \mathrm{H}, \mathrm{m})$, $7.40(1 \mathrm{H}, \mathrm{d}, J=7.5 \mathrm{~Hz}), 7.53(1 \mathrm{H}, \mathrm{d}, J=12.5 \mathrm{~Hz}), 8.15$
$(1 \mathrm{H}, \mathrm{s}), 8.28(1 \mathrm{H}, \mathrm{d}, J=7.5 \mathrm{~Hz}), 11.53-11.65(1 \mathrm{H}, \mathrm{br} \mathrm{s}$, NH). ${ }^{13} \mathrm{C}$ NMR (100 MHz, DMSO- $\left.d_{6}\right): 93.3,111.6,120.5$, $121.8(2 \mathrm{C}), 130.1,151.0(\mathrm{CH}), 118.0,126.1,136.5(\mathrm{C})$, $183.5(\mathrm{C}=\mathrm{O})$.

4.2.2. 1-Methyl-3-(3- $N, N$-dimethylamino-1-oxoprop-2enyl)-1 $\boldsymbol{H}$-indole 2 . Dimethylformamide-dimethylacetal $(45.0 \mathrm{~mL}, 339 \mathrm{mmol})$ was added to a solution of 3-acetylindole $(5.00 \mathrm{~g}, 31.4 \mathrm{mmol})$ in DMF $(63 \mathrm{~mL})$ and the solution was refluxed for $24 \mathrm{~h}$. Brine $(100 \mathrm{~mL})$ was added to the reaction mixture. After extraction with $\mathrm{CH}_{2} \mathrm{Cl}_{2}(3 \times 100 \mathrm{~mL})$, the combined organic fractions were dried over $\mathrm{MgSO}_{4}$ and concentrated under vacuum. The residue was dissolved in EtOAc $(50 \mathrm{~mL})$ and precipitated with cyclohexane $(350 \mathrm{~mL})$. The precipitate was collected by filtration and washed with $\mathrm{Et}_{2} \mathrm{O}(3 \times 20 \mathrm{~mL})$ to give $2(6.11 \mathrm{~g}$, $26.8 \mathrm{mmol}, 85 \%$ yield) as a pale yellow powder.

Mp $157-158^{\circ} \mathrm{C}$. IR (KBr) 3448, 1642, 1631, 1552, 1523, 1466, 1368, 1083. MS $m / z$ (EI) $228\left(\mathrm{M}^{+}\right) .{ }^{1} \mathrm{H}$ NMR $\left(400 \mathrm{MHz}\right.$, DMSO- $\left.d_{6}\right): 2.85-3.09\left(6 \mathrm{H}\right.$, br s, $\left.\mathrm{CH}_{3}\right), 3.83$ $\left(3 \mathrm{H}, \mathrm{s}, \mathrm{CH}_{3}\right), 5.71(1 \mathrm{H}, \mathrm{d}, J=12.5 \mathrm{~Hz}), 7.14(1 \mathrm{H}, \mathrm{t}$, $J=7.5 \mathrm{~Hz}), 7.20(1 \mathrm{H}, \mathrm{t}, J=7.0 \mathrm{~Hz}), 7.46(1 \mathrm{H}, \mathrm{d}, J=8.0 \mathrm{~Hz})$, $7.53(1 \mathrm{H}, \mathrm{d}, J=12.5 \mathrm{~Hz}), 8.15(1 \mathrm{H}, \mathrm{s}), 8.28(1 \mathrm{H}, \mathrm{d}$, $J=8.0 \mathrm{~Hz}) .{ }^{13} \mathrm{C}$ NMR $\left(100 \mathrm{MHz}, \mathrm{DMSO}-d_{6}\right): 32.8\left(\mathrm{CH}_{3}\right)$, $93.2,110.0,120.8,121.9(2 \mathrm{C}), 134.1,151.0(\mathrm{CH}), 116.9$, $126.5,137.1(\mathrm{C}), 183.0(\mathrm{C}=\mathrm{O})$.

4.2.3. 3-(2-Aminopyrimidin-4-yl)-1H-indole 3. Sodium methoxide (1.48 g, $27.4 \mathrm{mmol})$ and guanidine hydrochloride (788 mg, $8.25 \mathrm{mmol})$ were added to a solution of $1(1.15 \mathrm{~g}$, $5.37 \mathrm{mmol})$ in $i s o$-propanol $(6 \mathrm{~mL})$ and the mixture was refluxed for $48 \mathrm{~h}$. Water $(25 \mathrm{~mL})$ was added to the reaction mixture. After extraction with EtOAc $(3 \times 15 \mathrm{~mL})$, the organic fractions were dried over $\mathrm{MgSO}_{4}$ and concentrated under vacuum. The residue was dissolved in EtOAc $(5 \mathrm{~mL})$ and precipitated with cyclohexane $(25 \mathrm{~mL})$. The precipitate was collected by filtration and washed with $\mathrm{CH}_{2} \mathrm{Cl}_{2}(3 \times 5 \mathrm{~mL})$ to give 3 ( $831 \mathrm{mg}, 3.95 \mathrm{mmol}, 74 \%$ yield) as a beige powder.

Spectral data are in agreement with literature data. ${ }^{16}$

4.2.4. 3-(2-Amino-5-bromopyrimidin-4-yl)-1-methyl-1Hindole 4. Sodium methoxide $(5.79 \mathrm{~g}, 107 \mathrm{mmol})$ and guanidine hydrochloride $(3.84 \mathrm{~g}, 40.2 \mathrm{mmol})$ were added to a solution of $2(6.11 \mathrm{~g}, 26.8 \mathrm{mmol})$ in iso-propanol $(135 \mathrm{~mL})$ and the mixture was refluxed for $48 \mathrm{~h}$. Water $(250 \mathrm{~mL})$ was added to the reaction mixture. After extraction with EtOAc $(3 \times 150 \mathrm{~mL})$, the organic fractions were dried over $\mathrm{MgSO}_{4}$ and concentrated under vacuum. The residue was dissolved in EtOAc $(20 \mathrm{~mL})$ and precipitated with cyclohexane $(250 \mathrm{~mL})$. The precipitate was collected by filtration and washed with $\mathrm{CH}_{2} \mathrm{Cl}_{2}(3 \times 20 \mathrm{~mL})$ to give $4(2.64 \mathrm{~g}$, $11.8 \mathrm{mmol}, 44 \%$ yield) as a beige powder.

Mp 191-193 ${ }^{\circ} \mathrm{C}$. IR (KBr) 3454, 1624, 1576, 1534, 1458, 1219. HRMS $\left(\mathrm{ESI}^{+}\right)$calcd for $\mathrm{C}_{13} \mathrm{H}_{13} \mathrm{~N}_{4}(\mathrm{M}+\mathrm{H})^{+} 225.1140$, found 225.1130 . ${ }^{1} \mathrm{H}$ NMR $\left(400 \mathrm{MHz}, \mathrm{DMSO}-d_{6}\right): 3.85$ $(3 \mathrm{H}, \mathrm{s}), 6.37-6.45\left(2 \mathrm{H}\right.$, br s, $\left.\mathrm{NH}_{2}\right), 6.94(1 \mathrm{H}, \mathrm{d}, J=5.5 \mathrm{~Hz})$, $7.17\left(1 \mathrm{H}\right.$, ddd, $\left.J_{1}=8.0 \mathrm{~Hz}, J_{2}=7.0 \mathrm{~Hz}, J_{3}=1.0 \mathrm{~Hz}\right), 7.24$ $\left(1 \mathrm{H}, \mathrm{ddd}, J_{1}=8.0 \mathrm{~Hz}, J_{2}=7.0 \mathrm{~Hz}, J_{3}=1.5 \mathrm{~Hz}\right), 7.49(1 \mathrm{H}, \mathrm{d}$, $J=8.0 \mathrm{~Hz}), 8.10(1 \mathrm{H}, \mathrm{d}, J=5.5 \mathrm{~Hz}), 8.17(1 \mathrm{H}, \mathrm{s}), 8.59(1 \mathrm{H}$, d, $J=8.0 \mathrm{~Hz}) .{ }^{13} \mathrm{C}$ NMR $\left(100 \mathrm{MHz}\right.$, DMSO- $\left.d_{6}\right): 33.0$ 
$\left(\mathrm{CH}_{3}\right), 105.2,110.2,120.6,122.1,122.5,132.2,157.1(\mathrm{CH})$, $112.7,125.7,137.5,162.3,163.5(\mathrm{C})$.

4.2.5. 3-(2-Amino-5-bromopyrimidin-4-yl)- $1 \mathrm{H}$-indole 5 . NBS (372 mg, $2.09 \mathrm{mmol}$ ) was added to a solution of 3 (440 mg, $2.09 \mathrm{mmol})$ in THF $(18 \mathrm{~mL})$ and the reaction mixture was stirred at $0^{\circ} \mathrm{C}$ for $1.5 \mathrm{~h}$. After evaporation of the solvent, water was added to the reaction mixture and the solid was filtered and washed with water to give $\mathbf{5}$ (573 $\mathrm{mg}, 1.98 \mathrm{mmol}, 95 \%$ yield) as a red powder.

Mp $183-185^{\circ} \mathrm{C} . \quad$ IR $(\mathrm{KBr}) 3483,1654,1648,1638$, $1559,1542,1520,1460,1435$. HRMS $\left(\mathrm{ESI}^{+}\right)$calcd for $\mathrm{C}_{12} \mathrm{H}_{10}{ }^{79} \mathrm{BrN}_{4}(\mathrm{M}+\mathrm{H})^{+} 289.0089$, found 289.0097 . ${ }^{1} \mathrm{H}$ NMR $\left(400 \mathrm{MHz}\right.$, DMSO- $\left.d_{6}\right): 6.70-6.80(2 \mathrm{H}$, br s, $\left.\mathrm{NH}_{2}\right), 7.13\left(1 \mathrm{H}\right.$, ddd, $\left.J_{1}=8.0 \mathrm{~Hz}, J_{2}=7.0 \mathrm{~Hz}, J_{3}=1.0 \mathrm{~Hz}\right)$, $7.20\left(1 \mathrm{H}\right.$, ddd, $\left.J_{1}=8.0 \mathrm{~Hz}, J_{2}=7.0 \mathrm{~Hz}, J_{3}=1.0 \mathrm{~Hz}\right), 7.47$ $(1 \mathrm{H}, \mathrm{d}, J=8.0 \mathrm{~Hz}), 8.31(1 \mathrm{H}, \mathrm{s}), 8.48(1 \mathrm{H}, \mathrm{d}, J=3.0 \mathrm{~Hz})$, $8.54(1 \mathrm{H}, \mathrm{d}, J=8.0 \mathrm{~Hz}), 11.72-11.81(1 \mathrm{H}$, br s, NH). ${ }^{13} \mathrm{C}$ NMR (100 MHz, DMSO- $\left.d_{6}\right): 111.7,120.4,122.2$, $123.0,130.1,160.0(\mathrm{CH}), 102.1,111.8,126.3,136.0,159.2$, $161.8(\mathrm{C})$.

4.2.6. 3-(2-Amino-5-bromopyrimidin-4-yl)-1-methyl- $1 H$ indole 6. NBS ( $1.15 \mathrm{~g}, 6.46 \mathrm{mmol})$ was added to a solution of $4(1.45 \mathrm{~g}, 6.46 \mathrm{mmol})$ in THF $(65 \mathrm{~mL})$ and the reaction mixture was stirred at $0^{\circ} \mathrm{C}$ for $2 \mathrm{~h}$. Diethylether $(100 \mathrm{~mL})$ was added to the reaction mixture and the resulting precipitate was collected by filtration and washed with $\mathrm{Et}_{2} \mathrm{O}$ to give 6 ( $1.84 \mathrm{~g}, 6.07 \mathrm{mmol}, 94 \%$ yield $)$ as a brown powder.

Mp 211-213 ${ }^{\circ} \mathrm{C}$. IR (KBr) 3479, 1630, 1561, 1531, 1513 , 1450, 1370. HRMS (ESI ${ }^{+}$) calcd for $\mathrm{C}_{13} \mathrm{H}_{12}{ }^{79} \mathrm{BrN}_{4}$ $(\mathrm{M}+\mathrm{H})^{+}$303.0245, found 303.0228. ${ }^{1} \mathrm{H}$ NMR $(400 \mathrm{MHz}$, DMSO- $\left.d_{6}\right): 3.90\left(3 \mathrm{H}, \mathrm{s}, \mathrm{N}-\mathrm{CH}_{3}\right), 6.68-6.92(2 \mathrm{H}$, br s, $\left.\mathrm{NH}_{2}\right), 7.18(1 \mathrm{H}, \mathrm{t}, J=7.5 \mathrm{~Hz}), 7.27(1 \mathrm{H}, \mathrm{t}, J=7.5 \mathrm{~Hz}), 7.52$ $(1 \mathrm{H}, \mathrm{d}, J=8.0 \mathrm{~Hz}), 8.31(1 \mathrm{H}, \mathrm{s}), 8.53(1 \mathrm{H}, \mathrm{s}), 8.59(1 \mathrm{H}, \mathrm{d}$, $J=8.0 \mathrm{~Hz}) .{ }^{13} \mathrm{C}$ NMR $\left(100 \mathrm{MHz}\right.$, DMSO- $\left.d_{6}\right): 32.8(\mathrm{~N}-$ $\left.\mathrm{CH}_{3}\right), 109.8,120.5,122.1,123.0,133.9,159.5(\mathrm{CH})$, $101.6,110.5,126.5,136.3,158.7,161.4(\mathrm{C})$.

4.2.7. General procedure for the preparation of compounds 7-20. A solution of $\mathrm{Na}_{2} \mathrm{CO}_{3}$ (2.5 equiv) in $\mathrm{H}_{2} \mathrm{O}$ $(2 \mathrm{~mL} / \mathrm{mmol}$ ) was added to a suspension of 6 , the required boronic acid (1.1 equiv) and $\mathrm{Pd}\left(\mathrm{PPh}_{3}\right)_{4}(5 \mathrm{~mol} \%)$ in $\mathrm{EtOH}$ $(2 \mathrm{~mL} / \mathrm{mmol})$, and toluene $(2 \mathrm{~mL} / \mathrm{mmol})$. The resulting mixture was refluxed for $12 \mathrm{~h}$ under argon. The reaction mixture was extracted with $\mathrm{CHCl}_{3}(3 \times 20 \mathrm{~mL})$ and the combined organic layers were washed with $\mathrm{H}_{2} \mathrm{O}$ $(10 \mathrm{~mL}), 0.5 \mathrm{~N}$ aqueous $\mathrm{NaOH}(10 \mathrm{~mL}), \mathrm{H}_{2} \mathrm{O}(10 \mathrm{~mL})$, and brine $(10 \mathrm{~mL})$, and then dried over $\mathrm{MgSO}_{4}$ and concentrated under vacuum.

4.2.8. 3-(2-Amino-5-phenylpyrimidin-4-yl)- $1 \mathrm{H}$-indole 7 . Compound 7 was prepared according to the above general procedure, starting from $5(289 \mathrm{mg}, 1.00 \mathrm{mmol})$. The residue was dissolved in $\mathrm{Et}_{2} \mathrm{O}(2 \mathrm{~mL})$ and cyclohexane $(25 \mathrm{~mL})$ was added. The resulting precipitate was collected by filtration and further purified by flash chromatography (EtOAc/cyclohexane 8:2). The residue was dissolved in EtOAc $(2 \mathrm{~mL})$ and cyclohexane $(25 \mathrm{~mL})$ was added. The resulting precipitate was filtered to give 7 ( $59 \mathrm{mg}, 21 \%$ yield) as a beige powder.
Mp 226-228 ${ }^{\circ} \mathrm{C}$. IR (KBr) 3420, 3316, 1648, 1576, 1528, 1474. HRMS $\left(\mathrm{ESI}^{+}\right)$calcd for $\mathrm{C}_{18} \mathrm{H}_{15} \mathrm{~N}_{4}(\mathrm{M}+\mathrm{H})^{+}$ 287.1297, found 287.1299 . ${ }^{1} \mathrm{H}$ NMR $(400 \mathrm{MHz}$, DMSO$\left.d_{6}\right): 6.54-6.59\left(2 \mathrm{H}\right.$, br s, $\left.\mathrm{NH}_{2}\right), 6.60(1 \mathrm{H}, \mathrm{d}, J=3.0 \mathrm{~Hz})$, $7.06\left(1 \mathrm{H}\right.$, ddd, $\left.J_{1}=8.0 \mathrm{~Hz}, J_{2}=7.0 \mathrm{~Hz}, J_{3}=1.0 \mathrm{~Hz}\right), 7.12$ $\left(1 \mathrm{H}\right.$, ddd, $\left.J_{1}=8.0 \mathrm{~Hz}, J_{2}=7.0 \mathrm{~Hz}, J_{3}=1.5 \mathrm{~Hz}\right), 7.27-7.31$ $(2 \mathrm{H}, \mathrm{m}), 7.32-7.43(4 \mathrm{H}, \mathrm{m}), 8.00(1 \mathrm{H}, \mathrm{s}), 8.45(1 \mathrm{H}, \mathrm{d}$, $J=8.0 \mathrm{~Hz}), \quad 11.24-11.30(1 \mathrm{H}$, br $\mathrm{s}, \mathrm{NH}),{ }^{13} \mathrm{C}$ NMR $\left(100 \mathrm{MHz}, \mathrm{DMSO}-d_{6}\right): 111.4,120.0,121.9,122.8,127.1$, $128.8(3 \mathrm{C}), 129.5(2 \mathrm{C}), 157.9(\mathrm{CH}), 112.8,121.0,126.2$, $135.8,139.0,159.9,162.5(\mathrm{C})$.

4.2.9.3-(2-Amino-5-(4-acetylphenyl)pyrimidin-4-yl)- $H$ Hindole 8 . Compound $\mathbf{8}$ was prepared according to the above general procedure, starting from $5(289 \mathrm{mg}, 1.00 \mathrm{mmol})$. The residue was dissolved in EtOAc $(2 \mathrm{~mL})$ and cyclohexane $(25 \mathrm{~mL})$ was added. The resulting precipitate was collected by filtration and further purified by flash chromatography (EtOAc/cyclohexane 8:2). The residue was dissolved in EtOAc $(2 \mathrm{~mL})$ and cyclohexane $(25 \mathrm{~mL})$ was added. The resulting precipitate was filtered to give $8(90 \mathrm{mg}, 27 \%$ yield) as a pale yellow powder.

Mp 221-222 ${ }^{\circ} \mathrm{C}$. IR (KBr) 3502, 3313, 1676, 1627, 1603, $1584,1529,1508,1458,1269,1242$. HRMS $\left(\right.$ ESI $\left.^{+}\right)$calcd for $\mathrm{C}_{20} \mathrm{H}_{17} \mathrm{~N}_{4} \mathrm{O}(\mathrm{M}+\mathrm{H})^{+} 329.1402$, found 329.1403 . ${ }^{1} \mathrm{H}$ $\operatorname{NMR}\left(400 \mathrm{MHz}\right.$, DMSO- $\left.d_{6}\right): 2.59\left(3 \mathrm{H}, \mathrm{s}, \mathrm{CH}_{3}\right), 6.67-6.74$ $\left(2 \mathrm{H}\right.$, br s, $\left.\mathrm{NH}_{2}\right), 6.74(1 \mathrm{H}, \mathrm{d}, J=3.0 \mathrm{~Hz}), 7.05(1 \mathrm{H}$, ddd, $\left.J_{1}=8.0 \mathrm{~Hz}, J_{2}=7.0 \mathrm{~Hz}, J_{3}=1.0 \mathrm{~Hz}\right), 7.13\left(1 \mathrm{H}\right.$, ddd, $J_{1}=$ $\left.8.0 \mathrm{~Hz}, J_{2}=7.0 \mathrm{~Hz}, J_{3}=1.5 \mathrm{~Hz}\right), 7.35(1 \mathrm{H}, \mathrm{d}, J=8.0 \mathrm{~Hz})$, $7.44(2 \mathrm{H}, \mathrm{d}, J=8.5 \mathrm{~Hz}), 7.96(2 \mathrm{H}, \mathrm{d}, J=8.5 \mathrm{~Hz}), 8.07(1 \mathrm{H}$, s), $8.33(1 \mathrm{H}, \mathrm{d}, J=8.0 \mathrm{~Hz}), 11.26-11.34(1 \mathrm{H}$, br s, NH). ${ }^{13} \mathrm{C}$ NMR $\left(100 \mathrm{MHz}\right.$, DMSO- $\left.d_{6}\right): 26.7\left(\mathrm{CH}_{3}\right), 111.5$, $120.1,122.0,122.5,128.6(2 \mathrm{C}), 128.9,129.6(2 \mathrm{C}), 158.1$ $(\mathrm{CH}), 112.6,120.1,126.0,128.9,135.2,135.9,144.1$, $159.9,162.7(\mathrm{C}), 197.5(\mathrm{C}=\mathrm{O})$.

4.2.10. 3-(2-Amino-5-(4-biphenyl)pyrimidin-4-yl)- $1 H$-indole 9 . Compound 9 was prepared according to the above general procedure, starting from $5(289 \mathrm{mg}, 1.00 \mathrm{mmol})$. The residue was dissolved in EtOAc $(2 \mathrm{~mL})$ and cyclohexane $(25 \mathrm{~mL})$ was added. The solid was collected by filtration and further purified by flash chromatography (EtOAc/cyclohexane 8:2). The residue was dissolved in EtOAc $(2 \mathrm{~mL})$ and cyclohexane $(25 \mathrm{~mL})$ was added. The resulting precipitate was filtered to give 9 (178 mg, $49 \%$ yield) as a beige powder.

Mp $240-242^{\circ} \mathrm{C}$. IR (KBr) $3474,3406,1630,1576,1528$, 1457. HRMS $\left(\right.$ ESI $\left.^{+}\right)$calcd for $\mathrm{C}_{24} \mathrm{H}_{19} \mathrm{~N}_{4}(\mathrm{M}+\mathrm{H})^{+} 363.1610$, found $363.1606 .{ }^{1} \mathrm{H}$ NMR $\left(400 \mathrm{MHz}\right.$, DMSO- $\left.d_{6}\right)$ : 6.58$6.64\left(2 \mathrm{H}\right.$, br s, $\left.\mathrm{NH}_{2}\right), 6.75(1 \mathrm{H}, \mathrm{d}, J=3.0 \mathrm{~Hz}), 7.07(1 \mathrm{H}$, ddd, $\left.J_{1}=8.0 \mathrm{~Hz}, J_{2}=7.0 \mathrm{~Hz}, J_{3}=1,0 \mathrm{~Hz}\right), 7.13(1 \mathrm{H}$, ddd, $\left.J_{1}=8.0 \mathrm{~Hz}, J_{2}=7.0 \mathrm{~Hz}, J_{3}=1,5 \mathrm{~Hz}\right), 7.35(\mathrm{~d}, 1 \mathrm{H}, J=$ $8.0 \mathrm{~Hz}), 7.36-7.40(3 \mathrm{H}, \mathrm{m}), 7.48(2 \mathrm{H}, \mathrm{t}, J=7.5 \mathrm{~Hz}), 7.70$ $7.75(4 \mathrm{H}, \mathrm{m}), 8.06(1 \mathrm{H}, \mathrm{s}), 8.47(1 \mathrm{H}, \mathrm{d}, J=8.0 \mathrm{~Hz}), 11.27-$ $11.33\left(1 \mathrm{H}\right.$, br s, NH). ${ }^{13} \mathrm{C}$ NMR $\left(100 \mathrm{MHz}\right.$, DMSO- $\left.d_{6}\right):$ $111.5,120.0,121.9,122.8,126.5$ (2C), 126.9 (2C), 127.5, $128.9,129.0(2 \mathrm{C}), 130.0(2 \mathrm{C}), 158.0(\mathrm{CH}), 112.8,120.6$, $126.2,135.8,138.1,138.6,139.5,160.0,162.6(\mathrm{C})$.

4.2.11. 3-(2-Amino-5-(4-fluorophenyl)pyrimidin-4-yl)$1 \mathrm{H}$-indole 10. Compound 10 was prepared according to the above general procedure, starting from $5(289 \mathrm{mg}$, 
$1.00 \mathrm{mmol})$. The residue was dissolved in $\operatorname{EtOAc}(2 \mathrm{~mL})$ and cyclohexane $(25 \mathrm{~mL})$ was added. The resulting precipitate was collected by filtration and further purified by flash chromatography (EtOAc/cyclohexane 8:2). The residue was dissolved in EtOAc $(2 \mathrm{~mL})$ and cyclohexane $(25 \mathrm{~mL})$ was added. The resulting precipitate was filtered to give $\mathbf{1 0}$ (141 mg, 46\% yield) as a pale yellow powder.

Mp $232-234^{\circ} \mathrm{C}$. IR (KBr) $3410,1585,1526,1458,1426$, 1209. HRMS (ESI $\left.{ }^{+}\right)$calcd for $\mathrm{C}_{18} \mathrm{H}_{14} \mathrm{FN}_{4}(\mathrm{M}+\mathrm{H})^{+}$ 305.1202, found 305.1204. ' $\mathrm{H}$ NMR $(400 \mathrm{MHz}$, DMSO$\left.d_{6}\right): 6.56-6.62\left(2 \mathrm{H}, \mathrm{br} \mathrm{s}, \mathrm{NH}_{2}\right), 6.65(1 \mathrm{H}, \mathrm{d}, J=3.0 \mathrm{~Hz})$, $7.05\left(1 \mathrm{H}\right.$, ddd, $\left.J_{1}=8.0 \mathrm{~Hz}, J_{2}=7.0 \mathrm{~Hz}, J_{3}=1.0 \mathrm{~Hz}\right), 7.12$ $\left(1 \mathrm{H}\right.$, ddd, $\left.J_{1}=8.0 \mathrm{~Hz}, J_{2}=7.0 \mathrm{~Hz}, J_{3}=1.5 \mathrm{~Hz}\right), 7.19-7.26$ $(2 \mathrm{H}, \mathrm{m}), 7.29-7.37(3 \mathrm{H}, \mathrm{m}), 8.01(1 \mathrm{H}, \mathrm{s}), 8.40(1 \mathrm{H}, \mathrm{d}$, $J=8.0 \mathrm{~Hz}), \quad 11.25-11.34(1 \mathrm{H}$, br s, $\mathrm{NH}) .{ }^{13} \mathrm{C}$ NMR $\left(100 \mathrm{MHz}, \quad\right.$ DMSO- $\left.d_{6}\right): 111.5,115.7 \quad\left(\mathrm{~d}, \quad J_{\mathrm{CF}}=21 \mathrm{~Hz}\right)$, $120.1,122.0,122.7,128.8,131.5\left(\mathrm{~d}, J_{\mathrm{CF}}=8 \mathrm{~Hz}\right), 158.1$ $(\mathrm{CH}), 112.8,120.0,126.2,135.2\left(\mathrm{~d}, J_{\mathrm{CF}}=3 \mathrm{~Hz}\right), 135.8$, $160.1,161.5\left(\mathrm{~d}, J_{\mathrm{CF}}=243 \mathrm{~Hz}\right), 162.6(\mathrm{C})$.

4.2.12. 3-(2-Amino-5-(2,4-difluorophenyl)pyrimidin-4yl)-1H-indole 11. Compound 11 was prepared according to the above general procedure, starting from $5(289 \mathrm{mg}$, $1.00 \mathrm{mmol})$. The residue was dissolved in EtOAc $(2 \mathrm{~mL})$ and cyclohexane $(25 \mathrm{~mL})$ was added. The resulting precipitate was collected by filtration and further purified by flash chromatography (EtOAc/cyclohexane 8:2). The residue was dissolved in EtOAc $(2 \mathrm{~mL})$ and cyclohexane $(25 \mathrm{~mL})$ was added. The resulting precipitate was filtered to give $\mathbf{1 1}$ (110 mg, 34\% yield) as a beige powder.

Mp $222-224^{\circ} \mathrm{C}$. IR (KBr) 3509, 3409, 1607, 1587, 1530 , 1458, 1423, 1138. HRMS $\left(\mathrm{ESI}^{+}\right)$calcd for $\mathrm{C}_{18} \mathrm{H}_{13} \mathrm{~F}_{2} \mathrm{~N}_{4}$ $(\mathrm{M}+\mathrm{H})^{+} 323.1108$, found 323.1104 . ${ }^{1} \mathrm{H}$ NMR $(400 \mathrm{MHz}$, DMSO- $\left.d_{6}\right): 6.64(1 \mathrm{H}, \mathrm{d}, J=3.0 \mathrm{~Hz}), 6.67-6.73(2 \mathrm{H}$, br s, $\left.\mathrm{NH}_{2}\right), 7.09\left(1 \mathrm{H}\right.$, ddd, $\left.J_{1}=8.0 \mathrm{~Hz}, J_{2}=7.0 \mathrm{~Hz}, J_{3}=1.0 \mathrm{~Hz}\right)$, $7.14\left(1 \mathrm{H}\right.$, ddd, $\left.J_{1}=8.0 \mathrm{~Hz}, J_{2}=7.0 \mathrm{~Hz}, J_{3}=1.5 \mathrm{~Hz}\right), 7.20$ $\left(1 \mathrm{H}, \mathrm{dt}, J_{1}, \mathrm{CH}+\mathrm{CF}=8.5 \mathrm{~Hz}, J_{2}=2.5 \mathrm{~Hz}\right), 7.31\left(1 \mathrm{H}, \mathrm{dt}, J_{1}\right.$, $\left.\mathrm{CF}=9.5 \mathrm{~Hz}, J_{2}=2.5 \mathrm{~Hz}\right), 7.36(1 \mathrm{H}, \mathrm{d}, J=8.0 \mathrm{~Hz}), 7.45(1 \mathrm{H}$, $\left.\mathrm{dt}, J_{1}=8.5 \mathrm{~Hz}, J_{2}, \mathrm{CF}=7.0 \mathrm{~Hz}\right), 8.01(1 \mathrm{H}, \mathrm{s}), 8.52(1 \mathrm{H}, \mathrm{d}$, $J=8.0 \mathrm{~Hz}), \quad 11.24-11.36(1 \mathrm{H}$, br s, $\mathrm{NH}) .{ }^{13} \mathrm{C}$ NMR $\left(100 \mathrm{MHz}\right.$, DMSO- $\left.d_{6}\right): 104.5(\mathrm{t}, J=26 \mathrm{~Hz}), 111.5,112.2$ (dd, $\left.J_{\mathrm{CF} 1}=21 \mathrm{~Hz}, J_{\mathrm{CF} 2}=4 \mathrm{~Hz}\right), 120.1,122.1,122.9,127.6$, $133.2\left(\mathrm{dd}, J_{\mathrm{CF} 1}=10 \mathrm{~Hz}, J_{\mathrm{CF} 2}=4 \mathrm{~Hz}\right), 158.5(\mathrm{CH}), 112.9$, $113.3,122.8\left(\mathrm{dd}, J_{\mathrm{CF} 1}=17 \mathrm{~Hz}, J_{\mathrm{CF} 2}=4 \mathrm{~Hz}\right), 126.1,135.9$, $159.6\left(\mathrm{dd}, J_{\mathrm{CF} 1}=246 \mathrm{~Hz}, J_{\mathrm{CF} 2}=12 \mathrm{~Hz}\right), 160.7,162.0(\mathrm{dd}$, $\left.J_{\mathrm{CF} 1}=247 \mathrm{~Hz}, J_{\mathrm{CF} 2}=12 \mathrm{~Hz}\right), 163.0(\mathrm{C})$.

4.2.13. 3-(2-Amino-5-(4-trifluoromethylphenyl)pyrimidin-4-yl)-1H-indole 12. Compound 12 was prepared according to the above general procedure, starting from $\mathbf{5}$ $(289 \mathrm{mg}, 1.00 \mathrm{mmol})$. The residue was dissolved in EtOAc $(2 \mathrm{~mL})$ and cyclohexane $(25 \mathrm{~mL})$ was added. The resulting precipitate was collected by filtration and further purified by flash chromatography (EtOAc/cyclohexane 8:2). The residue was dissolved in EtOAc $(2 \mathrm{~mL})$ and cyclohexane $(25 \mathrm{~mL})$ was added. The resulting precipitate was filtered to give 12 (106 mg, 30\% yield) as a pale yellow powder.

Mp $125-127^{\circ} \mathrm{C}$. IR (KBr) 3484, 3365, 1636, 1615, 1585, $1528,1464,1439,1323,1172,1107,1068 . \operatorname{HRMS}\left(\mathrm{ESI}^{+}\right)$ calcd for $\mathrm{C}_{19} \mathrm{H}_{14} \mathrm{~F}_{3} \mathrm{~N}_{4}(\mathrm{M}+\mathrm{H})^{+} 355.1171$, found 355.1168 . ${ }^{1} \mathrm{H}$ NMR $\left(400 \mathrm{MHz}\right.$, DMSO- $\left.d_{6}\right): 6.68-6.75(2 \mathrm{H}$, br $\mathrm{s}$, $\left.\mathrm{NH}_{2}\right), 6.72(1 \mathrm{H}, \mathrm{d}, J=3.0 \mathrm{~Hz}), 7.05\left(1 \mathrm{H}, \mathrm{ddd}, J_{1}=8.0 \mathrm{~Hz}\right.$, $\left.J_{2}=7.0 \mathrm{~Hz}, J_{3}=1.0 \mathrm{~Hz}\right), 7.13\left(1 \mathrm{H}\right.$, ddd, $J_{1}=8.0 \mathrm{~Hz}, J_{2}=$ $\left.7.0 \mathrm{~Hz}, J_{3}=1.5 \mathrm{~Hz}\right), 7.35\left(1 \mathrm{H}, \mathrm{dt}, J_{1}=8.0 \mathrm{~Hz}, J_{2}=1.0 \mathrm{~Hz}\right)$, $7.52(2 \mathrm{H}, \mathrm{d}, J=8.0 \mathrm{~Hz}), 7.72(2 \mathrm{H}, \mathrm{d}, J=8.0 \mathrm{~Hz}), 8.09(1 \mathrm{H}$, s), $8.29(1 \mathrm{H}, \mathrm{d}, J=8.0 \mathrm{~Hz}), 11.30-11.37(1 \mathrm{H}$, br s, NH). ${ }^{13} \mathrm{C}$ NMR (100 MHz, DMSO- $\left.d_{6}\right): 111.5,120.0,122.0$, $122.4,125.5\left(2 \mathrm{C}, \mathrm{q}, J_{\mathrm{CF}}=4 \mathrm{~Hz}\right), 128.9,130.2(2 \mathrm{C}), 158.3$ $(\mathrm{CH}), 112.5,119.7,124.4\left(\mathrm{q}, J_{\mathrm{CF}}=272 \mathrm{~Hz}\right), 126.0,127.3$ $\left(\mathrm{q}, J_{\mathrm{CF}}=32 \mathrm{~Hz}\right), 135.9,143.3,159.9,162.8(\mathrm{C})$.

4.2.14. 3-(2-Amino-5-(4-trifluoromethoxyphenyl)pyrimidin-4-yl)- $1 \boldsymbol{H}$-indole 13 . Compound 12 was prepared according to the above general procedure, starting from 5 ( $289 \mathrm{mg}, 1.00 \mathrm{mmol}$ ). The residue was dissolved in EtOAc $(2 \mathrm{~mL})$ and cyclohexane $(25 \mathrm{~mL})$ was added. The resulting precipitate was collected by filtration and further purified by flash chromatography (EtOAc/cyclohexane $8: 2$ ). The residue was dissolved in EtOAc $(2 \mathrm{~mL})$ and cyclohexane $(25 \mathrm{~mL})$ was added. The resulting precipitate was filtered to give 13 (106 mg, 29\% yield) as a pale yellow powder.

Mp $104-106^{\circ} \mathrm{C}$. IR (KBr) $3485,3372,1627,1583,1535$, 1464, 1254, 1161. HRMS (ESI ${ }^{+}$) calcd for $\mathrm{C}_{19} \mathrm{H}_{14} \mathrm{~F}_{3} \mathrm{~N}_{4} \mathrm{O}$ $(\mathrm{M}+\mathrm{H})^{+} 371.1120$, found 371.1105 . ${ }^{1} \mathrm{H}$ NMR $(400 \mathrm{MHz}$, DMSO- $\left.d_{6}\right): 6.63-6.67\left(2 \mathrm{H}\right.$, br s, $\left.\mathrm{NH}_{2}\right), 6.70(1 \mathrm{H}, \mathrm{d}$, $J=3.0 \mathrm{~Hz}), 7.04\left(1 \mathrm{H}\right.$, ddd, $J_{1}=8.0 \mathrm{~Hz}, J_{2}=7.0 \mathrm{~Hz}, J_{3}=$ $1.0 \mathrm{~Hz}), 7.12\left(1 \mathrm{H}\right.$, ddd, $\left.J_{1}=8.0 \mathrm{~Hz}, J_{2}=7.0 \mathrm{~Hz}, J_{3}=1.5 \mathrm{~Hz}\right)$, $7.33-7.43(5 \mathrm{H}, \mathrm{m}), 8.06(1 \mathrm{H}, \mathrm{s}), 8.31(1 \mathrm{H}, \mathrm{d}, J=8.0 \mathrm{~Hz})$, $11.32-11.39$ (1H, br s, NH). ${ }^{13} \mathrm{C}$ NMR (100 MHz, DMSO$\left.d_{6}\right): 111.5,120.0,121.3(2 \mathrm{C}), 121.9,122.5,128.7,131.3$ $(2 \mathrm{C}), 158.1(\mathrm{CH}), 112.7,119.7,120.1\left(\mathrm{q}, J_{\mathrm{CF}}=256 \mathrm{~Hz}\right)$, $126.0,135.9,138.3,147.4\left(\mathrm{q}, J_{\mathrm{CF}}=2 \mathrm{~Hz}\right), 159.9,162.7(\mathrm{C})$.

4.2.15. 3-(2-A mino-5-phenylpyrimidin-4-yl)-1-methyl$\mathbf{H} \boldsymbol{H}$-indole 14 . Compound 14 was prepared according to the above general procedure, starting from $6(306 \mathrm{mg}$, $1.01 \mathrm{mmol})$. The residue was dissolved in EtOAc $(2 \mathrm{~mL})$ and cyclohexane $(25 \mathrm{~mL})$ was added. The resulting precipitate was collected by filtration and washed with $\mathrm{Et}_{2} \mathrm{O}$ $(3 \times 2 \mathrm{~mL})$ to give $14(133 \mathrm{mg}, 0.443 \mathrm{mmol}, 44 \%$ yield) as a pale yellow powder.

Mp $210-212^{\circ} \mathrm{C}$. IR (KBr) 3489, 1625, 1583, 1528, 1459 , 1370. HRMS $\left(\mathrm{ESI}^{+}\right)$calcd for $\mathrm{C}_{19} \mathrm{H}_{17} \mathrm{~N}_{4}(\mathrm{M}+\mathrm{H})^{+}$ 301.1453, found 301.1438. ${ }^{1} \mathrm{H}$ NMR $(400 \mathrm{MHz}$, DMSO$\left.d_{6}\right): 3.58\left(3 \mathrm{H}, \mathrm{s}, \mathrm{CH}_{3}-\mathrm{N}\right), 6.56-6.60\left(2 \mathrm{H}, \mathrm{br} \mathrm{s}, \mathrm{NH}_{2}\right), 6.63$ $(1 \mathrm{H}, \mathrm{d}, J=3.0 \mathrm{~Hz}), 7.07\left(1 \mathrm{H}\right.$, ddd, $J_{1}=8.0 \mathrm{~Hz}, J_{2}=7.0 \mathrm{~Hz}$, $\left.J_{3}=1.0 \mathrm{~Hz}\right), \quad 7.18 \quad\left(1 \mathrm{H}, \quad\right.$ ddd, $J_{1}=8.0 \mathrm{~Hz}, \quad J_{2}=7.0 \mathrm{~Hz}$, $\left.J_{3}=1.0 \mathrm{~Hz}\right), 7.26-7.30(2 \mathrm{H}, \mathrm{m}), 7.35-7.42(4 \mathrm{H}, \mathrm{m}), 8.02$ $(1 \mathrm{H}, \mathrm{s}), 8.32(1 \mathrm{H}, \mathrm{d}, J=8.0 \mathrm{~Hz}) .{ }^{13} \mathrm{C}$ NMR $(100 \mathrm{MHz}$, DMSO $\left.-d_{6}\right): 32.8\left(\mathrm{CH}_{3}-\mathrm{N}\right), 109.8,120.2,121.9,122.8$, $127.1,128.8(2 \mathrm{C}), 129.3(2 \mathrm{C}), 132.6,158.1(\mathrm{CH}), 112.1$, $121.0,126.5,136.4,138.7,159.5,162.5(\mathrm{C})$.

4.2.16. 3-(2-Amino-5-(4-acetylphenyl)pyrimidin-4-yl)-1methyl- $\mathbf{H}$-indole $\mathbf{1 5}$. Compound 15 was prepared according to the above general procedure, starting from 6 ( $289 \mathrm{mg}$, $0.95 \mathrm{mmol})$. The residue was dissolved in EtOAc $(2 \mathrm{~mL})$ and $\mathrm{Et}_{2} \mathrm{O}(25 \mathrm{~mL})$ was added. The resulting precipitate was collected by filtration and washed with $\mathrm{Et}_{2} \mathrm{O}(3 \times 2 \mathrm{~mL})$ to give 15 (192 mg, $0.56 \mathrm{mmol}, 59 \%$ yield) as an orange powder. 
Mp 201-203 ${ }^{\circ} \mathrm{C}$. IR (KBr) 3402, 1654, 1578, 1528, 1508, $1478,1459,1364$. HRMS $\left(\mathrm{ESI}^{+}\right)$calcd for $\mathrm{C}_{21} \mathrm{H}_{19} \mathrm{~N}_{4} \mathrm{O}$ $(\mathrm{M}+\mathrm{H})^{+}$343.1559, found 343.1566. ${ }^{1} \mathrm{H}$ NMR $(400 \mathrm{MHz}$, DMSO- $\left.d_{6}\right): 2.59\left(3 \mathrm{H}, \mathrm{s}, \mathrm{CH}_{3}\right), 3.63\left(3 \mathrm{H}, \mathrm{s}, \mathrm{N}-\mathrm{CH}_{3}\right), 6.68-$ $6.74\left(2 \mathrm{H}\right.$, br s, $\left.\mathrm{NH}_{2}\right), 6.85(1 \mathrm{H}, \mathrm{s}), 7.05(1 \mathrm{H}, \mathrm{t}, J=7.5 \mathrm{~Hz})$, $7.18(1 \mathrm{H}, \mathrm{t}, J=7.5 \mathrm{~Hz}), 7.41(1 \mathrm{H}, \mathrm{d}, J=8.5 \mathrm{~Hz}), 7.44(2 \mathrm{H}$, d, $J=8.0 \mathrm{~Hz}), 7.94(2 \mathrm{H}, \mathrm{d}, J=8.0 \mathrm{~Hz}), 8.09(1 \mathrm{H}, \mathrm{s}), 8.16$ $(1 \mathrm{H}, \mathrm{d}, J=8.0 \mathrm{~Hz}) .{ }^{13} \mathrm{C}$ NMR $\left(100 \mathrm{MHz}\right.$, DMSO- $\left.d_{6}\right): 26.7$ $\left(\mathrm{CH}_{3}\right), 32.8\left(\mathrm{CH}_{3}-\mathrm{N}\right), 109.9,120.2,122.0,122.4,128.6$ (2C), $129.3(2 \mathrm{C}), 132.7,158.4(\mathrm{CH}), 111.9,120.0,126.3$, $135.1,136.5,143.7,159.4,162.7(\mathrm{C}), 197.5(\mathrm{C}=\mathrm{O})$.

4.2.17. 3-(2-Amino-5-(4-biphenyl)pyrimidin-4-yl)-1methyl-1H-indole 16 . Compound 16 was prepared according to the above general procedure, starting from 6 $(289 \mathrm{mg}, 0.95 \mathrm{mmol}$ ). The residue was dissolved in EtOAc $(2 \mathrm{~mL})$ and $\mathrm{Et}_{2} \mathrm{O}(25 \mathrm{~mL})$ was added. The resulting precipitate was collected by filtration and washed with $\mathrm{Et}_{2} \mathrm{O}$ $(3 \times 2 \mathrm{~mL})$ to give $16(168 \mathrm{mg}, 0.45 \mathrm{mmol}, 47 \%$ yield $)$ as an orange powder.

Mp 210-212 ${ }^{\circ} \mathrm{C}$. IR (KBr) 3490, 1624, 1578, 1528, 1478, 1459 , 1364. HRMS $\left(\mathrm{ESI}^{+}\right)$calcd $\mathrm{C}_{25} \mathrm{H}_{21} \mathrm{~N}_{4}(\mathrm{M}+\mathrm{H})^{+}$ 377.1766, found 377.1747 . ${ }^{1} \mathrm{H}$ NMR $(400 \mathrm{MHz}$, DMSO$\left.d_{6}\right): 3.60\left(3 \mathrm{H}, \mathrm{s}, \mathrm{N}-\mathrm{CH}_{3}\right), 6.60-6.65\left(2 \mathrm{H}, \mathrm{br} \mathrm{s}, \mathrm{NH}_{2}\right), 6.82$ $(1 \mathrm{H}, \mathrm{s}), 7.07(1 \mathrm{H}, \mathrm{t}, J=7.5 \mathrm{~Hz}), 7.18(1 \mathrm{H}, \mathrm{t}, J=7.5 \mathrm{~Hz})$, $7.35-7.42(4 \mathrm{H}, \mathrm{m}), 7.48(2 \mathrm{H}, \mathrm{t}, J=7.5 \mathrm{~Hz}), 7.68-7.74(4 \mathrm{H}$, $\mathrm{m}), 8.09(1 \mathrm{H}, \mathrm{s}), 8.31(1 \mathrm{H}, \mathrm{d}, J=8.0 \mathrm{~Hz}) .{ }^{13} \mathrm{C} \mathrm{NMR}$ $\left(100 \mathrm{MHz}, \mathrm{DMSO}-d_{6}\right): 32.8\left(\mathrm{~N}-\mathrm{CH}_{3}\right), 109.9,120.2,121.9$, $122.8,126.5$ (2C), 126.9 (2C), $127.5,129.0$ (2C), 129.8 (2C), 132.7, $158.3(\mathrm{CH}), 112.1,120.5,126.5,136.5,137.8$, $138.6,139.6,159.5,162.5(\mathrm{C})$.

4.2.18. 3-(2-Amino-5-(4-fluorophenyl)pyrimidin-4-yl)-1methyl-1H-indole 17 . Compound 17 was prepared according to the above general procedure, starting from 6 ( $289 \mathrm{mg}, 0.95 \mathrm{mmol}$ ). The residue was dissolved in EtOAc $(2 \mathrm{~mL})$ and $\mathrm{Et}_{2} \mathrm{O}(25 \mathrm{~mL})$ was added. The resulting precipitate was collected by filtration and washed with $\mathrm{Et}_{2} \mathrm{O}$ $(3 \times 2 \mathrm{~mL})$ to give $17(156 \mathrm{mg}, 0.49 \mathrm{mmol}, 52 \%$ yield) as a pale brown powder.

Mp 222-224 ${ }^{\circ} \mathrm{C}$; IR (KBr) 3489, 1629, 1581, 1535, 1478, 1460. HRMS (ESI $\left.{ }^{+}\right)$calcd for $\mathrm{C}_{19} \mathrm{H}_{16} \mathrm{FN}_{4}(\mathrm{M}+\mathrm{H})^{+}$ 319.1359, found 319.1359. ${ }^{1} \mathrm{H}$ NMR $(400 \mathrm{MHz}$, DMSO$\left.d_{6}\right): 3.63\left(3 \mathrm{H}, \mathrm{s}, \mathrm{N}-\mathrm{CH}_{3}\right), 6.58-6.64\left(2 \mathrm{H}, \mathrm{br} \mathrm{s}, \mathrm{NH}_{2}\right), 6.72$ $(1 \mathrm{H}, \mathrm{s}), 7.07\left(1 \mathrm{H}\right.$, ddd, $\left.J_{1}=8.0 \mathrm{~Hz}, J_{2}=7.0 \mathrm{~Hz}, J_{3}=1.0 \mathrm{~Hz}\right)$, 7.16-7.24 (3H, m), 7.28-7.33 $(2 \mathrm{H}, \mathrm{m}), 7.40(1 \mathrm{H}, \mathrm{d}$, $J=8.0 \mathrm{~Hz}), 8.03(1 \mathrm{H}, \mathrm{s}), 8.28(1 \mathrm{H}, \mathrm{d}, J=8.0 \mathrm{~Hz}) .{ }^{13} \mathrm{C}$ NMR $\left(100 \mathrm{MHz}\right.$, DMSO- $\left.d_{6}\right): 32.8\left(\mathrm{~N}-\mathrm{CH}_{3}\right), 109.9,115.7$ $\left(\mathrm{d}, J_{\mathrm{CF}}=21 \mathrm{~Hz}\right), 120.2,122.0,122.7,131.3\left(\mathrm{~d}, J_{\mathrm{CF}}=8 \mathrm{~Hz}\right)$,

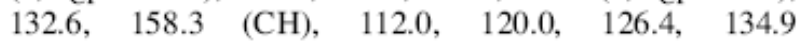
$\left(\mathrm{d}, J_{\mathrm{CF}}=3 \mathrm{~Hz}\right), 136.5,159.6,161.4\left(\mathrm{~d}, J_{\mathrm{CF}}=244 \mathrm{~Hz}\right)$, $162.6(\mathrm{C})$.

4.2.19. 3-(2-Amino-5-(2,4-difluorophenyl)pyrimidin-4yl)-1-methyl-1H-indole 18 . Compound 18 was prepared according to the above general procedure, starting from 6 ( $289 \mathrm{mg}, 0.95 \mathrm{mmol}$ ). The residue was dissolved in EtOAc $(2 \mathrm{~mL})$ and $\mathrm{Et}_{2} \mathrm{O}(25 \mathrm{~mL})$ was added. The resulting precipitate was collected by filtration and washed with $\mathrm{Et}_{2} \mathrm{O}$ $(3 \times 2 \mathrm{~mL})$ to give $18(68 \mathrm{mg}, 0.202 \mathrm{mmol}, 21 \%$ yield $)$ as a pale yellow powder.
$\mathrm{Mp}>235^{\circ} \mathrm{C} . \mathrm{IR}(\mathrm{KBr}) 3490,1629,1583,1542,1534,1478$, 1459. HRMS $\left(\mathrm{ESI}^{+}\right)$calcd for $\mathrm{C}_{19} \mathrm{H}_{15} \mathrm{~F}_{2} \mathrm{~N}_{4}(\mathrm{M}+\mathrm{H})^{+} 337.1265$, found $337.1258 .{ }^{1} \mathrm{HNMR}\left(500 \mathrm{MHz}, \mathrm{DMSO}-d_{6}\right): 3.63(3 \mathrm{H}, \mathrm{s}$, $\left.\mathrm{N}-\mathrm{CH}_{3}\right), 6.67(1 \mathrm{H}, \mathrm{s}), 6.69-6.75\left(2 \mathrm{H}\right.$, br s, $\left.\mathrm{NH}_{2}\right), 7.12(1 \mathrm{H}$, ddd, $\left.J_{1}=8.0 \mathrm{~Hz}, J_{2}=7.0 \mathrm{~Hz}, J_{3}=1.0 \mathrm{~Hz}\right), 7.17-7.23(2 \mathrm{H}$, m), $7.29\left(1 \mathrm{H}, \mathrm{dt}, J_{1, \mathrm{CF}}=9.5 \mathrm{~Hz}, J_{2}=2.5 \mathrm{~Hz}\right), 7.41(1 \mathrm{H}, \mathrm{d}$, $J=8.0 \mathrm{~Hz}), 7.44\left(1 \mathrm{H}, \mathrm{dt}, J_{1}=8.5 \mathrm{~Hz}, J_{2, \mathrm{CF}}=7.0 \mathrm{~Hz}\right), 8.01$ $(1 \mathrm{H}, \mathrm{s}), 8.45(1 \mathrm{H}, \mathrm{d}, J=8.0 \mathrm{~Hz}) .{ }^{13} \mathrm{C}$ NMR $(125 \mathrm{MHz}$, DMSO- $\left.d_{6}\right): 32.8\left(\mathrm{~N}-\mathrm{CH}_{3}\right), 104.5\left(\mathrm{t}, J_{\mathrm{CF}}=26 \mathrm{~Hz}\right), 109.9$, $112.2\left(\mathrm{dd}, J_{\mathrm{CF} 1}=21 \mathrm{~Hz}, J_{\mathrm{CF} 2}=3 \mathrm{~Hz}\right), 120.4,122.1,122.9$, $131.5,133.1\left(\mathrm{dd}, J_{\mathrm{CF} 1}=10 \mathrm{~Hz}, J_{\mathrm{CF} 2}=4 \mathrm{~Hz}\right), 158.8(\mathrm{CH})$, $112.2,113.4,122.6\left(\mathrm{dd}, J_{\mathrm{CF} 1}=16 \mathrm{~Hz}, J_{\mathrm{CF} 2}=4 \mathrm{~Hz}\right), 126.4$, $136.5,159.5\left(\mathrm{dd}, J_{\mathrm{CF} 1}=246 \mathrm{~Hz}, J_{\mathrm{CF} 2}=12 \mathrm{~Hz}\right), 160.3,162.0$ $\left(\mathrm{dd}, J_{\mathrm{CF} 1}=246 \mathrm{~Hz}, J_{\mathrm{CF} 2}=12 \mathrm{~Hz}\right), 163.0(\mathrm{C})$.

4.2.20. 3-(2-Amino-5-(4-trifluoromethylphenyl)pyrimidin-4-yl)-1-methyl-1H-indole 19 . Compound 19 was prepared according to the above general procedure, starting from 6 ( $289 \mathrm{mg}, 0.95 \mathrm{mmol})$. The residue was dissolved in $\mathrm{Et}_{2} \mathrm{O}(2 \mathrm{~mL})$ and cyclohexane $(25 \mathrm{~mL})$ was added. The resulting precipitate was collected by filtration and washed with cyclohexane $(3 \times 2 \mathrm{~mL})$ to give 19 (105 mg, $0.285 \mathrm{mmol}, 30 \%$ yield) as a pale orange powder.

Mp $185-187^{\circ} \mathrm{C}$. IR (KBr) $3449,1637,1582,1542,1528$, $1478,1466,1323,1107$. HRMS $\left(\mathrm{ESI}^{+}\right)$calcd for $\mathrm{C}_{20} \mathrm{H}_{16} \mathrm{~N}_{4} \mathrm{~F}_{3}(\mathrm{M}+\mathrm{H})^{+}$369.1327, found 369.1317. ${ }^{1} \mathrm{H}$ NMR $\left(400 \mathrm{MHz}, \mathrm{DMSO}-d_{6}\right): 3.64\left(3 \mathrm{H}, \mathrm{s}, \mathrm{N}-\mathrm{CH}_{3}\right), 6.68-6.76$ $\left(2 \mathrm{H}\right.$, br s, $\left.\mathrm{NH}_{2}\right), 6.87(1 \mathrm{H}, \mathrm{s}), 7.04\left(1 \mathrm{H}\right.$, ddd, $J_{1}=8.0 \mathrm{~Hz}$, $\left.J_{2}=7.0 \mathrm{~Hz}, \quad J_{3}=1.0 \mathrm{~Hz}\right), \quad 7.17 \quad\left(1 \mathrm{H}, \quad\right.$ ddd, $J_{1}=8.0 \mathrm{~Hz}$, $\left.J_{2}=7.0 \mathrm{~Hz}, J_{3}=1.0 \mathrm{~Hz}\right), 7.41(1 \mathrm{H}, \mathrm{d}, J=8.0 \mathrm{~Hz}), 7.51(2 \mathrm{H}$, d, $J=8.0 \mathrm{~Hz}), 7.69(2 \mathrm{H}, \mathrm{d}, J=8.0 \mathrm{~Hz}), 8.07(1 \mathrm{H}, \mathrm{d}$, $J=8.0 \mathrm{~Hz}), 8.12(1 \mathrm{H}, \mathrm{s}) .{ }^{13} \mathrm{C}$ NMR $\left(100 \mathrm{MHz}, \mathrm{DMSO}-d_{6}\right)$ : $32.8\left(\mathrm{CH}_{3}-\mathrm{N}\right), 110.0,120.2,122.0,122.3,125.5(2 \mathrm{C}, \mathrm{q}$, $\left.J_{\mathrm{CF}}=4 \mathrm{~Hz}\right), 129.9(2 \mathrm{C}), 132.7,158.6(\mathrm{CH}), 111.9,119.7$, $124.4\left(\mathrm{q}, J_{\mathrm{CF}}=272 \mathrm{~Hz}\right), 126.2,127.3\left(\mathrm{q}, J_{\mathrm{CF}}=32 \mathrm{~Hz}\right)$, $136.6,143.1,159.5,162.7(\mathrm{C})$.

4.2.21. 3-(2-Amino-5-(4-trifluoromethoxyphenyl)pyrimidin-4-yl)-1-methyl-1H-indole 20. Compound 20 was prepared according to the above general procedure, starting from 6 ( $289 \mathrm{mg}, 0.95 \mathrm{mmol})$. The residue was dissolved in EtOAc $(2 \mathrm{~mL})$ and $\mathrm{Et}_{2} \mathrm{O}(25 \mathrm{~mL})$ was added. The resulting precipitate was collected by filtration and washed with $\mathrm{Et}_{2} \mathrm{O}(3 \times 2 \mathrm{~mL})$ to give $\mathbf{2 0}(218 \mathrm{mg}, 0.57 \mathrm{mmol}, 60 \%$ yield $)$ as a brown powder.

Mp $183-185^{\circ} \mathrm{C}$. IR (KBr) 3487, 1624, 1526, 1476, 1456, $1258,1217,1195,1178$. HRMS $\left(\mathrm{ESI}^{+}\right)$calcd for $\mathrm{C}_{20} \mathrm{H}_{16} \mathrm{~F}_{3} \mathrm{~N}_{4} \mathrm{O}(\mathrm{M}+\mathrm{H})^{+}$385.1276, found 385.1273. ${ }^{1} \mathrm{H}$ NMR $\left(400 \mathrm{MHz}, \mathrm{DMSO}-d_{6}\right): 3.63\left(3 \mathrm{H}, \mathrm{s}, \mathrm{N}-\mathrm{CH}_{3}\right), 6.62-6.68$ $\left(2 \mathrm{H}\right.$, br s, $\left.\mathrm{NH}_{2}\right), 6.75(1 \mathrm{H}, \mathrm{s}), 7.04(1 \mathrm{H}, \mathrm{t}, J=7.5 \mathrm{~Hz}), 7.18$ $(1 \mathrm{H}, \mathrm{t}, J=7.5 \mathrm{~Hz}), 7.34-7.42(5 \mathrm{H}, \mathrm{m}), 8.09(1 \mathrm{H}, \mathrm{s}), 8.13$ $(1 \mathrm{H}, \mathrm{d}, J=8.0 \mathrm{~Hz}) .{ }^{13} \mathrm{C}$ NMR $\left(100 \mathrm{MHz}, \mathrm{DMSO}-d_{6}\right): 32.7$ $\left(\mathrm{N}-\mathrm{CH}_{3}\right), 109.9,120.2,121.4(2 \mathrm{C}), 121.9,122.4,131.2$ (2C), 132.6, $158.3(\mathrm{CH}), 112.0,119.6,120.1(\mathrm{q}, J=256 \mathrm{~Hz})$, $126.2,136.5,138.1,147.4\left(\mathrm{q}, J_{\mathrm{CF}}=2 \mathrm{~Hz}\right), 159.5,162.6(\mathrm{C})$.

\section{Acknowledgements}

The authors thank the European Union Prokinase Research Consortium for financial support. 


\section{References and notes}

1. Jiang, X.; Zhao, B.; Britton, R.; Lim, L. Y.; Leong, D.; Sanghera, J. S.; Zhou, B.-B. S.; Piers, E.; Andersen, R. J.; Roberge, M. Mol. Cancer Ther. 2004, 3, 1221.

2. Hénon, H.; Messaoudi, S.; Anizon, F.; Aboab, B.; Kucharczyk, N.; Léonce, S.; Golsteyn, R. M.; Pfeiffer, B.; Prudhomme, M. Eur. J. Pharmacol. 2007, 554, 106.

3. Meijer, L.; Raymond, E. Acc. Chem. Res. 2003, 36, 417.

4. Eisenbrand, G.; Hippe, F.; Jakobs, S.; Muehlbeyer, S. J. Cancer Res. Clin. Oncol. 2004, 130, 627.

5. Sassatelli, M.; Bouchikhi, F.; Messaoudi, S.; Anizon, F.; Debiton, E.; Barthomeuf, C.; Prudhomme, M.; Moreau, P. Eur. J. Med. Chem. 2006, $41,88$.

6. Tamaoki, T.; Nomoto, H.; Takahashi, I.; Kato, Y.; Morimoto, M.; Tomita, F. Biochem. Biophys. Res. Commun. 1986, 135, 397.

7. Omura, S.; Sasaki, Y.; Iwai, Y.; Takeshima, H.J. Antibiot. 1995, 48,535 .

8. Mizuno, K.; Saido, T. C.; Ohno, S.; Tamaoki, T.; Suzuki, K. FEBS Lett. 1993, 330, 114.

9. Nakanishi, S.; Matsuda, Y.; Iwahashi, K.; Kase, H. J. Antibiot. 1986, 34, 1066.

10. Toullec, D.; Pianetti, P.; Coste, H.; Bellevergue, P.; GrandPerret, T.; Ajakane, M.; Baudet, V.; Boissin, P.; Boursier, E.; Loriolle, F.; Duhamel, L.; Charon, D.; Kirilovsky, J. J. Biol. Chem. 1991, 266, 15771.

11. Davis, P. D.; Hill, C. H.; Keech, E.; Lawton, G.; Nixon, J. S.; Sedgwick, A. D.; Wadsworth, J.; Westmacott, D.; Wilkinson, S. E. FEBS Lett. 1989, 259, 61.

12. Davis, P. D.; Hill, C. H.; Lawton, G. Eur. Patent EP0328026 A1, 1989; Chem. Abstr. 1990, 112, 98378.

13. Franco, L. H.; Bal de Kier Joffé, E.; Puricelli, L.; Tatian, M.; Seldes, A. M.; Palermo, J. A. J. Nat. Prod. 1998, 61, 1130.

14. Franco, L. H.; Palermo, J. A. Chem. Pharm. Bull. 2003, 51, 975.

15. Gompel, M.; Leost, M.; Bal de Kier Joffé, E.; Puricelli, L.; Franco, L. H.; Palermo, J.; Meijer, L. Bioorg. Med. Chem. Lett. 2004, 14, 1703.

16. Radwan, M. A. A.; El-Sherbiny, M. Bioorg. Med. Chem. 2007, $14,1206$.

17. Huang, S.; Li, R.; Connolly, P. J.; Emanuel, S.; FuentesPesquera, A.; Adams, M.; Gruninger, R. H.; Seraj, J.; Middleton, S. A.; Davis, J. M.; Moffat, D. F. C. Bioorg. Med. Chem. Lett. 2007, 17, 2179.

18. Fischer, P. M.; Wang, S.; Meades, C. K.; Andrews, M. J. I.; Gibson, D.; Duncan, K. PCT Int. Appl. WO 2006075152 Al, 2006; Chem. Abstr. 2006, 145, 167275.

19. Ratcliffe, A. J.; Alam, M.; Beevers, R. E.; Davenport, R. J.; Davies, N.; Haughan, A. F.; Jones, M. W.; Lowe, C.; Perry, B. G.; Phillips, D. J.; Pitt, W. R.; Sharpe, A. PCT Int. Appl. WO 2006038001 Al, 2006; Chem. Abstr. 2006, 144, 390934.

20. Bressi, J. C.; Gangloff, A. R.; Hosfield, D. J.; Jennings, A. J.; Paraselli, B. R.; Stafford, J. A. PCT Int. Appl. WO 2005123672 A2, 2005; Chem. Abstr. 2006, 144, 88168.

21. Reddy, E. P.; Reddy, M. V. R.; Cosenza, S. C.; Gumireddy, K. PCT Int. Appl. WO 2005065074 A2, 2005; Chem. Abstr. 2005, $143,149136$.

22. Agarwal, A.; Srivastava, K.; Puri, S. K.; Chauhan, P. M. S. Bioorg. Med. Chem. Lett. 2005, 15, 3133.

23. Agarwal, A.; Kumar, B.; Mehrotra, P. K.; Chauhan, P. M. S. Bioorg. Med. Chem. 2005, 13, 1893.
24. Bollbuck, B.; Denholm, A.; Eder, J.; Hersperger, R.; Janser, P.; Revesz, L.; Schlapbach, A.; Waelchli, R. PCT Int. Appl. WO 2004089913 Al, 2004; Chem. Abstr. 2004, 141, 379931.

25. Kidwai, M.; Rastogi, S.; Saxena, S. Bull. Korean Chem. Soc. 2003, 24, 1575.

26. Kim, Y.; Hanney, B. PCT Int. Appl. WO 2002102783, 2002; Chem. Abstr. 2003, 138, 55974.

27. Batchelor, M. J.; Moffat, D. F. C.; Davis, J. M.; Hutchings, M. C. PCT Int. Appl. WO 2000078731 Al, 2000; Chem. Abstr. 2001, 134, 71598.

28. Berger, J.; Flippin, L. A.; Greenhouse, R.; Jaime-Figueroa, S.; Liu, Y.; Miller, A. K.; Putman, D. G.; Weinhardt, K. K.; Zhao, S.-H. PCT Int. Appl. WO 9744326 A1, 1997; Chem. Abstr. $1998,128,48235$.

29. Davis, P. D.; Moffat, D. F. C.; Davis, J. M.; Hutchings, M. C. PCT Int. Appl. WO 9719065 Al, 1997; Chem. Abstr. 1997, $127,81461$.

30. Zimmerman, J.; Buchdunger, E.; Mett, H.; Meyer, T.; Lydon, N. B. Bioorg. Med. Chem. Lett. 1997, 7, 187.

31. Zimmerman, J.; Caravatti, G.; Mett, H.; Meyer, T.; Mueller, M.; Lydon, N. B.; Fabbro, D. Arch. Pharm. (Weinheim, Ger.) $1996,329,371$.

32. Zimmerman, J. PCT Int. Appl. WO 9509847 Al, 1995; Chem. Abstr. 1995, 123, 313996.

33. Zimmerman, J. PCT Int. Appl. WO 9509852 A1, 1995; Chem. Abstr. 1995, 123, 169650.

34. Paul, R.; Hallett, W. A.; Hanifin, J. W.; Reich, M. F.; Johnson, B. D.; Lenhard, R. H.; Dusza, J. P.; Kerwar, S. S.; Lin, Y.-I.; Pickett, W. C.; Seifert, C. M.; Torley, L. W.; Tarrant, M. E.; Wrenn, S. J. Med. Chem. 1993, 36, 2716.

35. Torley, L. W.; Johnson, B. B.; Dusza, J. P. Eur. Pat. Appl. EP 233461 A2, 1987; Chem. Abstr. 1988, 108, 112478.

36. Kobayashi, G.; Matsuda, Y. Jpn Tokyo Koho JP 46008698, 1971; Chem. Abstr. 1971, 75, 449129.

37. Kobayashi, G.; Furukawa, S.; Matsuda, Y.; Washida, Y. Yakugaku Zasshi 1967, 87, 857.

38. Davies, S. P.; Reddy, H.; Caivano, M.; Cohen, P. Biochem. J. 2000, 351, 95 .

39. Bain, J.; McLauchlan, H.; Elliott, M.; Cohen, P. Biochem. J. 2003, 371, 199.

40. http://www.upstate.com/features/kp_reference.asp.

41. Peifer, C.; Stoiber, T.; Unger, E.; Totzke, F.; Schächtele, C.; Marmé, D.; Brenk, R.; Klebe, G.; Schollmeyer, D.; Dannhardt, G. J. Med. Chem. 2006, 49, 1271.

42. Dow, R. L. Curr. Med. Chem. 1994, 1, 192.

43. Jiang, B.; Yang, C.-G. Heterocycles 2000, 53, 1489.

44. Fresneda, P. M.; Molina, P.; Delgado, S.; Bleda, J. A. Tetrahedron Lett. 2000, 41, 4777.

45. Fresneda, P. M.; Molina, P.; Bleda, J. A. Tetrahedron 2001, 57, 2355.

46. Karpov, A. S.; Merkul, E.; Rominger, F; Müller, T. J. J. Angew. Chem., Int. Ed. 2005, 44, 6951.

47. Fresneda, P. M.; Delgado, S.; Francesch, A.; Manzanares, I.; Cuevas, C.; Molina, P. J. Med. Chem. 2006, 49, 1217.

48. Bergman, J.; Rehn, S. Tetrahedron 2002, 58, 9179.

49. Stanovnik, B.; Svete, J. Chem. Rev. 2004, 104, 2433.

50. Meissner, A.; Sørensen, W. Magn. Reson. Chem. 2001, 39, 49.

51. Byth, K. F.; Culshaw, J. D.; Green, S.; Oakes, S. E.; Thomas, A. P. Bioorg. Med. Chem. Lett. 2004, 14, 2245.

52. Hannah, D. R.; Sherer, E. C.; Davies, R. V.; Titman, R. B.; Laughton, C. A.; Stevens, M. F. G. Bioorg. Med. Chem. 2000, 8,739. 